Yu.V. Shurub

\title{
STATISTICAL OPTIMIZATION OF FREQUENCY REGULATED INDUCTION ELECTRIC DRIVES WITH SCALAR CONTROL
}

\begin{abstract}
Purpose. Working out of technique of synthesizing statistically optimal controllers of induction electric drives with frequency scalar control operating under stochastic loads. Methodology. It is shown, that one of the ways of increasing the energy efficiency of induction electric drives at random changes of loads is to create closed systems of electric drives with statistically optimal regulators that would take into account the stochastic nature of the disturbances and acted as filters of occasional high frequency fluctuations. The structure of such controls depends on the statistical characteristics of stochastic loads - correlation function and spectral density. In this mode, the minimum loss in dynamic mode with random intensive changed loads is provided by criterion of minimum mean square error of optimal parameter control. In the case of frequency controlled electric drive such a parameter is the optimum rotor flux. Results. The optimal structures of regulators of electric drives under stochastic loads according to type of random disturbances are identified. Originality. The technique of statistically optimal synthesis with feature of the expansion of a random process load on average, served as a useful signal, and high-frequency fluctuations around the average value, served as barrier, is developed. Practical value. On an example of simulation of work of the electric drive of a crusher of grain it is shown efficiency of a filtration by statically optimal regulator of the high-frequency components of random torques of load, reduction of dispersions of its outlet parameters, increase of cyclic indicators of energy efficiency of the electric drive, such as cyclic efficiency and power factor. References 4, tables 1, figures 3.
\end{abstract}

Key words: induction electric drive, frequency scalar control, stochastic load, optimal regulator.

Разработана методика синтеза статистически оптимальных регуляторов асинхронных электроприводов с частотным скалярным управлением, работающих при стохастических моментах нагрузки. Определень оптимальные структуры регуляторов электроприводов такого класса в зависимости от вида случайных возмущений. На примере моделирования работы электропривода дробилки зерна показана эффективность фильтрации статистически оптимальным регулятором высокочастотных составляющих моментов нагрузки и повышение циклических показателей энергоэффективности электропривода, таких как цикловые КПД и коэффициент мощцности. Библ. 4, табл. 1, рис. 3.

Ключевые слова: асинхронный электропривод, частотное скалярное управление, случайная нагрузка, оптимальный регулятор.

Introduction. Wide class of induction electric drives including those used in agricultural mechanisms, in housing, in construction have load torque changing at random laws. They are drives of mechanisms as grain crushers, shredders, granulators, mixers, conveyors and more. At random changes since the current electric load, speed and electromagnetic torque of induction motor (IM) are also changed by random laws.

Stochastic character changes perturbation of electric greatly impairs their performance, requires overstatement installed capacity of motors, causing shocks in the mechanical parts of the drive makes the current surge in the motor and networks that worsens the quality of energy consumption and increases power loss, reduced quality regulation drives increases the likelihood of overload induction motor and its failure, makes it impossible for the realization of optimal modes of energy consumption in electric closed systems, such as «frequency converterinduction motor», «voltage converter - induction motor».

The goal of the work is to develop methods of statistically optimum synthesis of regulators of induction electric drives with frequency converters at random perturbations for mechanisms that do not require speed control.
Problem definition. One of the ways of increasing the energy efficiency of induction electric drives at random character of changes of loads is to create closed systems of electric statistically optimal regulators that would take into account the stochastic nature of the disturbances and acted as filters occasional high load fluctuations. The structure of such controls depends on the statistical characteristics of stochastic loads correlation function and spectral density.

The problem of optimizing energy consumption in closed induction electric drive with frequency converters at a static moment load is sufficiently developed in Ukraine and abroad [1-3]. At the same time poorly investigated processes in such systems in dynamic mode at a variable for process cycle time of loading, including random law. In this mode, the minimum loss in dynamic mode with loads of intensive change at random by law, meets the criterion of minimum mean square error of optimal parameter adjustment. In the case of frequency controlled electric drive such parameter is the optimum rotor flux. This can be considered two types of frequency control of IM - scalar and vector.

(C) Yu.V. Shurub 
When vector control possible direct regulation of the rotor flux through the separation process and flow control point. But high quality filtration components load depends on the quality of stabilization flux path that is difficult to implement frequency converters with voltage source properties because of the electromagnetic connection between circuits regulating moment and flux [4].

Based on these reasons, this work as an example of the synthesis of statistically optimal system is considered «frequency converter - induction motor» (FC - IM) with scalar control at a constant preset speed.

We know that in order to ensure minimum power losses in the induction motor by changing the static load should be regulated optimal flux linkage, which is defined by the formula

$$
\Psi_{\text {opt }}=\Psi_{n} \frac{I_{2}}{I_{2 n}} \sqrt{\frac{\Delta P_{\text {var }_{n}}}{I_{\mu n}^{2} R_{1}+\Delta P_{\text {const }_{n}}\left(\frac{f}{f_{n}}\right)^{\beta}}},
$$

where $\Delta P_{\text {const }_{n}}$ are the nominal losses in motor steel; $\Delta P_{\mathrm{var}_{n}}$ are the nominal losses in motor copper; $\Psi_{n}$ is the nominal motor rotor flux linkage; $I_{\mu n}$ is the nominal magnetization current; $I_{2 n}, I_{2}$ are the nominal and current value of the rotor currents; $f_{n}, f$ are the nominal and current value of the frequency of power; $\beta$ is the coefficient depending on the steel grade (here we accept $1.5) ; R_{1}$ is the stator resistance.

For an optimal flux linkage (1) at a given speed of rotation, defined preset frequency, you must submit the optimum voltage $U_{\text {opt }}$ that work in the area of slip can be determined approximately equivalent circuit IM by the formula

$$
U_{o p t}=\Psi_{o p t} f+I_{1} \sqrt{R_{1}^{2}+\left(2 \pi f L_{1}\right)^{2}} .
$$

This can be done using functional circuit closed system «frequency converter - induction motor», which is shown in Fig. 1. In this diagram marked: IM asynchronous motor, $\mathrm{CD}$ - computing device in which the calculated value $U_{o p t}, \mathrm{FC}$ - frequency converter, VS voltage sensor, CS - current sensor, VR - voltage regulator, $f_{z}$ - speed signal setting.

Incoming control action of the motor as part of a closed system «frequency converter - induction motor» mode optimizing the law is $\Psi=\Psi_{\text {opt }}$ stator voltage and frequency, which are connected to each other by (2). This frequency control circuit stabilizes the speed at a given level, and voltage regulation circuit stabilizes the optimum flux.

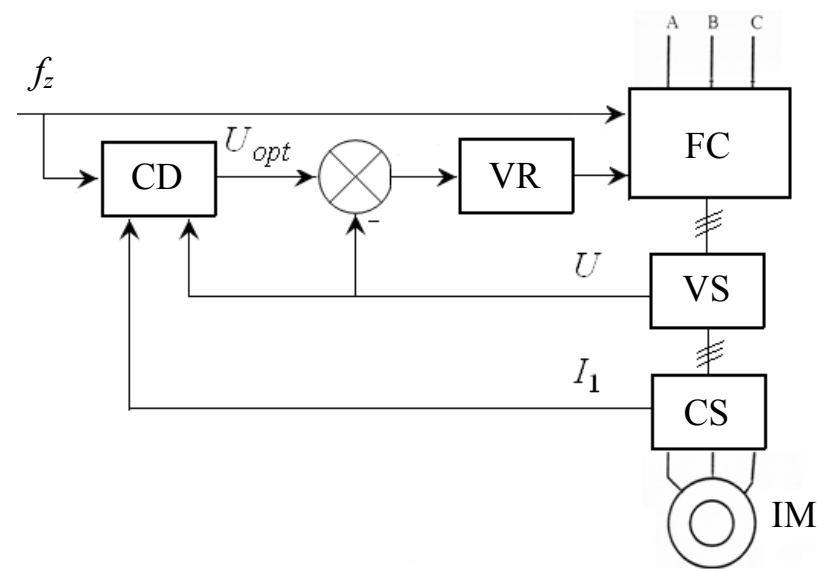

Fig. 1. Functional scheme of the closed system

«frequency converter - induction motor» at scalar control

At static load torque the motor will run with minimal loss of power provided the relationships (1) and (2). In dynamic mode at a dramatically changing the law for random load electric current, electromagnetic torque and speed IM and replaced by random laws. Moreover, the stator and rotor currents at the given voltage and frequency and assuming the work of the linear section of the magnetization curve is uniquely determined slip. With the dynamic changes sliding computing device as a result of electromagnetic and electromechanical inertia will respond to the change of load inertia that will not receive optimal treatment in every time. So for implementation mode power optimization requires stabilization of sliding (rotor speed) at stochastic dynamic loads.

In scalar control only amplitude adjustable rotor flux vector using the ratio of $U / f$ and its phase is unmanageable. Because of this electromagnetic torque is unmanaged components that do not allow to get high quality regulation in electric drives with variable control signals such as tracking, positional drives, but provides a fairly high quality of stabilization speed at variable loads, therefore, frequency regulated electric drives with scalar control can provide power optimization mode.

Results of investigations. Linearized block diagram of electric system «frequency converter - induction motor» of the scalar control is shown in Fig. 2.

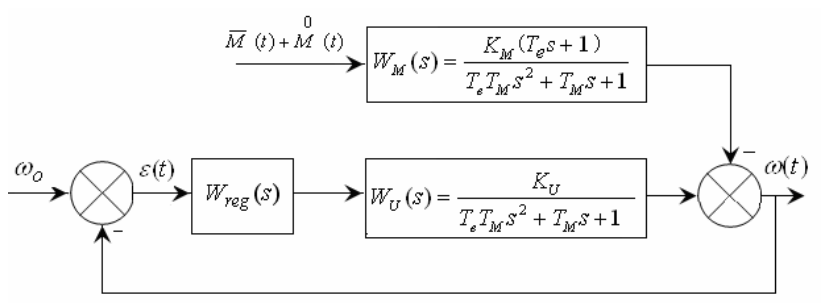

Fig. 2. Block diagram of the electric drive

In this diagram: $\omega_{o}$ is the setting task (cyclic load speed); $\omega(t)$ is the electric drive adjustable output value (cyclic speed); $M(t)=\bar{M}(t)+\stackrel{0}{M(t)}$ is the random perturbation signal that served as the sum of the mean 
$\bar{M}(t)$ and centered random process $M(t), W_{\text {reg }}(s)$ is the transfer function of the regulator, $W_{U}(s)$ is the transfer function motor control signal, $W_{M}(s)$ is the transfer function motor disturbance (torque of resistance).

In the transfer function in Fig. 2 marked: $s$ is the Laplace operator, $T_{e}$ is the electromagnetic time constant, $T_{M}$ is the electromechanical time constant of the induction motor, $K_{U}$ is the transfer coefficient for signal control; $K_{M}$ is the transfer coefficient for the disturbance.

Input signal of the regulator

$$
\varepsilon(t)=\omega_{o}-\omega(t)
$$

we call accidental error system.

For optimality criterion we take the minimum criteria rms random error adjustment $\varepsilon_{\text {rms }}$ which in the case of stationary and ergodicity process load can be calculated as the square root of the average error of regulation in time $\bar{\varepsilon}^{2}$ for a sufficiently long period of observation $T \rightarrow \propto$ :

$$
\varepsilon_{r m s}=\sqrt{\bar{\varepsilon}^{2}}=\sqrt{\lim _{T \rightarrow \infty} \int_{0}^{T} \varepsilon^{2}(t) d t} \rightarrow \min .
$$

This paper discusses the problem of statistically optimal synthesis for arbitrary structure regulator, which is previously unknown. This problem is formulated as follows. By according is the statistical characteristics of the signal disturbance - load $M(t)$ such as its spectral density $S_{M}(\omega)$, transfer function and object management asynchronous electric drive. Need to find a transfer function regulator $W_{\text {reg }}(s)$ which provides the minimum mean square error of the system $\varepsilon_{\mathrm{rms}}$.

For centered stationary random process with zero expectation root mean square error equal to the variance of the random error system

$$
\varepsilon_{r m s}=\sqrt{\bar{\varepsilon}^{2}}=\sqrt{D_{\varepsilon}},
$$

which can be found on the basis of the laws of conversion statistical characteristics of random process induction electric closed system of transfer function for the disturbance

$$
W(s)=\frac{W_{M}(s)}{1+W_{\text {reg }}(s) \cdot W_{U}(s)} .
$$

In accordance with the Hinchyn-Wiener law

$$
D_{\varepsilon}=\int_{0}^{\infty}\left|\frac{W_{M}(j \omega)}{1+W_{r e g}(j \omega) \cdot W_{U}(j \omega)}\right|^{2} S_{M}(\omega) d \omega,
$$

where $W_{U}(j \omega), W_{M}(j \omega)$ are the frequency transfer function for the electric drive control and disturbance, respectively; $W_{\text {reg }}(j \omega)$ is the frequency transfer function of the regulator.

So, in order to minimize $\varepsilon_{\text {rms }}$, it is necessary that the condition $D_{\varepsilon} \rightarrow$ min should be correct.

Because of the complexity of (7) directly used for synthesis of optimal statistical it can not. Therefore solution of this problem was the technique of statistically optimal synthesis feature is the expansion of a random process load on average $\bar{M}(t)$ served as a useful signal and high-frequency fluctuations around the average value 0 $M(t)$ served as barrier - centered stationary random process.

After expansion signal load on the signal $\bar{M}(t)$ and 0 signal of interference $M(t)$ dynamic random error system can be represented as the difference of two output signals (Fig. 3), one of which is designed with the terms of optimizing energy consumption at the moment of static frequency transfer function of a closed system disturbance

$$
k_{0}(j \omega)=\frac{W_{M}(j \omega)}{1+W_{U}(j \omega)},
$$

and the frequency transfer function of the second system

$$
k(j \omega)=\frac{W_{M}(j \omega)}{1+W_{r e g}(j \omega) \cdot W_{U}(j \omega)}
$$

is still unknown because the unknown frequency transfer function regulator $W_{\text {reg }}(j \omega)$ which sought to ensure the conditions of minimum mean square error by filtering high frequency fluctuations.

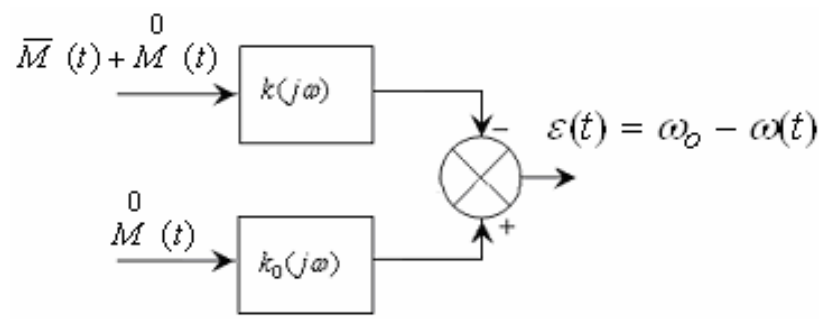

Fig. 3. Block diagram for optimal synthesis

Let $S_{\bar{M}}(\omega)$ is the spectral density of the useful signal and $S_{M}(\omega)$ is the noise signal spectral density.

Then the spectral density of random dynamic error adjustment according to Fig. 3

$$
\begin{aligned}
& S_{\varepsilon}(\omega)=\left|k_{0}(j \omega)-k(j \omega)\right|^{2} \cdot S_{\bar{M}}(\omega)+ \\
& +|k(j \omega)|^{2} \cdot S_{M}(\omega) .
\end{aligned}
$$

The dispersion of accidental error of regulation

$$
\begin{aligned}
& D_{\varepsilon}=\int_{-\infty}^{\infty}\left|k_{0}(j \omega)\right|^{2} \cdot\left\{\left|1-k^{\prime}(j \omega)\right|^{2} \cdot S_{\bar{M}}(\omega)+\right. \\
& \left.+\left|k^{\prime}(j \omega)\right|^{2} \cdot S_{M}(\omega)\right\} d \omega,
\end{aligned}
$$

where $k^{\prime}(j \omega)=\frac{k(j \omega)}{k_{0}(j \omega)}$.

After some changes and minimize the expression (11) we obtain the frequency transfer function statistically optimal system would be: 


$$
k_{\text {opt }}(j \omega)=\frac{k_{0}(j \omega) \cdot S_{\bar{M}}(\omega)}{S_{\bar{M}}(\omega)+S_{M}(\omega)} .
$$

Analysis of the frequency transfer function (12) for loads with exponential and exponentially-cosine correlation functions shown that it has positive poles of the variable $j \omega$, therefore, such a system can not be physically realized. To correct solution of this problem should be made of the frequency transfer function that has negative poles, and the other part rejected.

For this it is necessary from $K_{o p t}(j \omega)$ to separate the part $k_{\text {opt }}^{\text {real }}(j \omega)$ that is physically realized.

For this first we expand the denominator of (12) in the complex multipliers

$$
S_{\bar{M}}(\omega)+S_{M}(\omega)=\psi(j \omega) \cdot \psi(-j \omega),
$$

where $\psi(j \omega)$ is the function which all poles by complex variable $j \omega$ are negative; $\psi(-j \omega)$ is the function which all poles by complex variable $j \omega$ are positive.

Now we divide the function $\frac{S_{\bar{M}}(\omega)}{\psi(-j \omega)}$ into two components:

$$
\frac{S_{\bar{M}}(\omega)}{\psi(-j \omega)}=\left\{\frac{S_{\bar{M}}(\omega)}{\psi(-j \omega)}\right\}^{+}+\left\{\frac{S_{\bar{M}}(\omega)}{\psi(-j \omega)}\right\}^{-},
$$

where $\left\{\frac{S_{\bar{M}}(\omega)}{\psi(-j \omega)}\right\}^{+}$is the component which has negative poles of the complex variable $j \omega$ and can be realized physically, $\left\{\frac{S_{\bar{M}}(\omega)}{\psi(-j \omega)}\right\}^{-}$is the component which has positive poles of the complex variable $j \omega$ and can not be realized physically.

Rejecting the part of the function $\frac{S_{\bar{M}}(\omega)}{\psi(-j \omega)}$ that can not be realized physically

We obtain closest to the optimum frequency transfer function of the closed electric drive that can be physically realized:

$$
k_{\text {opt }}^{r e a l}(j \omega)=\frac{k_{0}(j \omega)}{\psi(j \omega)} \cdot\left\{\frac{S_{\bar{M}_{c}}(\omega)}{\psi(-j \omega)}\right\}^{+} .
$$

For obtaining transfer function of statistically optimal system $k_{\text {opt }}^{\text {real }}(s)$ It is necessary to present (15) as a ratio of complex variable polynomials $j \omega$ with following substitution of the variable $j \omega$ by the operator $s$.

After determining the transfer function statistically optimal system $k_{\text {opt }}^{\text {real }}(s)$ we determine transfer function of the regulator with the following relationship which can be obtained from (9) solving this equation relatively $W_{\text {reg }}(s)$ :

$$
W_{\text {reg }}(s)=\frac{k_{\text {opt }}^{\text {real }}(s)-W_{M}(s)}{k_{\text {opt }}^{r e a l}(s) \cdot W_{U}(s)} .
$$

Calculations showed that under load, which is exponential correlation function $R(\tau)=D e^{-\alpha|\tau|}$ by statistically optimal transfer function of the regulator will be consistent connection aperiodic link and forcing a firstorder link $W_{r e g}(s)=\frac{a_{0} s+a_{1}}{b_{0} s+b_{1}}$, and for loading of exponentially-cosine correlation function $R(\tau)=D e^{-\alpha|\tau|} \cos \beta \tau-$ serial aperiodic link and forcing a second-order link $W_{\text {reg }}(s)=\frac{a_{0} s+a_{1}}{b_{0} s^{2}+b_{1} s+b_{2}}$. Factors data gear functions are functions of the parameters of gear function electric $W_{U}(s)$ and $W_{M}(s)$ and parameters of correlation functions load $R(\tau)$.

Investigations of modes of induction electric drives with random character changes the load carried by the example of simulation of electric uniflow mills grain type of induction motor 4A80V2 load which has exponentially-cosine correlation function that corresponds to the statistical characteristics of the realizations obtained experimentally.

Simulation of the application of electric statistically optimal regulator showed reduction of variance electric output parameters (electromagnetic torque, current, speed) by $50-70 \%$.

Rate energy reserves through the use of statistically optimal regulator may cycle using indicators such as cycle efficiency and power factor. These figures for electric uniflow mills grain given in Table 1 in the case of nonregulated electric drive, the electric drive system «frequency converter - induction motor» with a typical speed control, designed with a static mode (with PI regulator) and with statistically optimal regulator.

Table 1

Power indicators of electric drives

\begin{tabular}{|c|c|c|}
\hline Drive type & Cycle efficiency & $\cos \varphi$ \\
\hline Non-regulated & $70 \%$ & 0.64 \\
\hline With PI regulator & $71 \%$ & 0.68 \\
\hline With statically optimal regulator & $76 \%$ & 0.81 \\
\hline
\end{tabular}

From Table 1 it is shown that the use of default PI regulator does not allow to get significant energy efficiency through the use of optimal interlinkage law regulation in terms of action stochastic dynamic loads due to impacts of electromagnetic and electromechanical inertia. The use of statistically optimal regulator with stochastic disturbances enhanced the performance of electric energy. Moreover, the scalar driving these figures are close to the values obtained by statistical optimization 
is electric vector control [4] under the same load conditions, but at simplest terms technical implementation.

\section{Conclusions.}

1. At actions of dynamic stochastic loads for optimal control law implementing it is appropriate to use statistically optimal regulators instead of or together with standard regulators.

2. Simulation of electric drive of the grain crusher showed statistically optimum filtration efficiency by the regulator of high-frequency components of random points in the load, improving cycle power performance of electric drive.

\section{REFERENCES}

1. E. Levi, M. Sokola, A. Boglietti, M. Pastorelli. Iron loss in rotor-flux oriented induction machines: identification, assessment of detuning, and compensation. IEEE Transactions on Power Electronics, 1996, vol.11, no.5, pp. 698-709. doi: 10.1109/63.535402.

2. Dymko S., Peresada S., Leidhold R. Torque control of saturated induction motors with torque per ampere ratio

How to cite this article:

Shurub Yu.V. Statistical optimization of frequency regulated induction electric drives with scalar control. Electrical engineering \& electromechanics, 2017, no.1, pp. 26-30. doi: 10.20998/2074-272X.2017.1.05. maximization. Proceedings of 2014 IEEE International Conference on Intelligent Energy and Power Systems, 2-6 June 2014, Kyiv, Ukraine, pp. 251-256.

3. Braslavskii I.Ya., Ishmatov Z.Sh., Plotnikov Yu.V. Energyand resource-conserving technologies based on controllable asynchronous drives. Russian Electrical Engineering, 2004, vol.75, no.9, pp. 30-36.

4. Shurub Yu.V., Dudnyk A.O., Lavinskiy D.S. Optimization of regulators of frequency controlled induction electric drives under the stochastic loadings. Tekhnichna elektrodynamika, 2016, no.4, pp. 53-55. (Ukr).

Received 25.10.2016

Yu.V. Shurub, Candidate of Technical Science,

The Institute of Electrodynamics of the NAS of Ukraine, 56, prospekt Peremogy, Kiev-57, 03680, Ukraine, phone+38 044 3662637, e-mail: shurub@bigmir.net 